\title{
Magnetocaloric Effect in $\mathrm{NdNi}_{4} \mathrm{Si}$ Compound
}

\author{
M. Falkowski*, T. Toliński And A. KowalczyK \\ Institute of Molecular Physics, Polish Academy of Sciences, M. Smoluchowskiego 17, 60-179 Poznań, Poland
}

\begin{abstract}
On the basis of the thermodynamic approach, we report the magnetocaloric properties of the ternary ferromagnetic $\mathrm{NdNi}_{4} \mathrm{Si}$ compound with magnetic phase transition temperature $T_{\mathrm{C}}$ at $8 \mathrm{~K}$. The saturated magnetic moment in $H=9 \mathrm{~T}$ is equal to $1.5 \mu_{\mathrm{B}} / \mathrm{f}$.u. at $4.2 \mathrm{~K}$ and the compound crystallizes in the hexagonal $\mathrm{CaCu}_{5}$-type structure (space group $P 6 / \mathrm{mmm}$ ). The magnetocaloric effect was calculated in terms of the isothermal magnetic entropy change $\Delta S_{\mathrm{M}}$ as well as the adiabatic temperature change $\Delta T_{\mathrm{ad}}$ using the specific heat data and magnetization measurements. Within the second order phase transition significant values of these parameters have been observed.
\end{abstract}

PACS: $75.30 . \mathrm{Sg}, 75.30 . \mathrm{Kz}, 75.47 . \mathrm{Np}, 75.40 .-\mathrm{s}$

\section{Introduction}

Since many years a lot of attention has been dedicated to extensive investigations of the magnetocaloric effect (MCE) in various magnetic intermetallic compounds due to the possibility of the development of the alternative and eco-friendly cooling technologies. MCE belongs to the variety of magnetic phenomena appearing in intermetallic compounds formed between the rare earths $(\mathrm{R})$ and the transition metal (TM) and attracting attention owing to their potential applications [1-4]. The phenomenon of MCE in a general way is defined by the isothermal entropy change $\Delta S_{\mathrm{M}}$ and the adiabatic temperature change $\Delta T_{\mathrm{ad}}$ of the magnetic materials under the influence of an external magnetic field. The magnetic refrigeration based on the MCE is advantageous over the vapor cooling as it can be more efficient and environmentally benign.

The ternary $\mathrm{RNi}_{4} \mathrm{Si}$ compounds, where $\mathrm{R}$ is a rare earth were previously investigated mainly in respect of the crystal structure, magnetic and electronic properties. $\mathrm{RNi}_{4} \mathrm{Si}$ compounds crystallize in the hexagonal $\mathrm{CaCu}_{5}$ -type structure. Apart from La, Ce, Pr and $\mathrm{Yb}$, these compounds order ferromagnetically with $T_{\mathrm{C}}$ in the range from $5.7 \mathrm{~K}(\mathrm{Tm})$ to $22 \mathrm{~K}(\mathrm{Gd})$. The $T_{\mathrm{C}}$ values of $\mathrm{RNi}_{4} \mathrm{Si}$ follow the de Gennes function for heavy rare earths [5].

For $\mathrm{NdNi}_{4} \mathrm{Si}$ the magnetic phase transition occurs at $T_{\mathrm{C}}=8 \mathrm{~K}$ and the saturation magnetic moment is $1.5 \mu_{\mathrm{B}} /$ f.u. at $4.2 \mathrm{~K}$ in $H=9 \mathrm{~T}$ [6]. The increased values of the electronic heat coefficient $\gamma=85 \mathrm{~mJ} \mathrm{~mol}^{-1} \mathrm{~K}^{-2}$ is not related to the heavy fermion state but means only that there are other contributions in this temperature range, like: spin fluctuations near $T_{\mathrm{C}}$, crystal field, disorder, etc. In the low-temperature dependence of the

\footnotetext{
* corresponding author; e-mail: falkowski@ifmpan.poznan.pl
}

specific heat for $\mathrm{NdNi}_{4} \mathrm{Si}$ and for zero-field, $C_{p}(T)$ shows a maximum near $T_{\mathrm{C}}$ and a well-defined $\lambda$-type anomaly is visible. The externally applied magnetic fields lead to the quenching and shifting the peak in the vicinity of $T_{\mathrm{C}}$ to higher temperatures, typical for ferromagnets [6].

We have investigated the MCE effect on the basis of the specific heat and the magnetization measurements for $\mathrm{NdNi}_{4} \mathrm{Si}$ compound, which exhibits relatively large value of $\Delta S_{\mathrm{M}}$ around the magnetic ordering temperature.

\section{Experimental details}

The sample was obtained by induction melting of the elements in an argon atmosphere. The preparation details of the polycrystalline sample of $\mathrm{NdNi}_{4} \mathrm{Si}$ were described in previous paper [6]. The heat capacity measurements were carried out on the Quantum Design PPMS platform in the temperature range of 1.9-300 K in magnetic fields up to $9 \mathrm{~T}$ by the relaxation method using the two- $\tau$ model. The magnetization curves were also measured on the PPMS system using the VSM option.

\section{Results and discussion}

The several isothermal magnetization curves measured around the transition temperature are plotted in Fig. 1. The magnetic isotherms were collected on increasing and decreasing field up to $9 \mathrm{~T}$ and in the temperature range from 2 to $60 \mathrm{~K}$. The hysteresis was not observed for $T<T_{\mathrm{C}}$. The field dependence of magnetization below the transition temperature shows the ferromagnetic nature of $\mathrm{NdNi}_{4} \mathrm{Si}$, whereas above $T_{\mathrm{C}}$, especially above about $25 \mathrm{~K}$ the $M(H)$ dependence is linear.

To deduce the nature of the magnetic transition, we have plotted the Arrot plots presented in Fig. 2. The positive slope and the absence of the S-shaped curves for $M^{2}$ as a function of $H / M$ support the second order nature of the magnetic phase transition. 


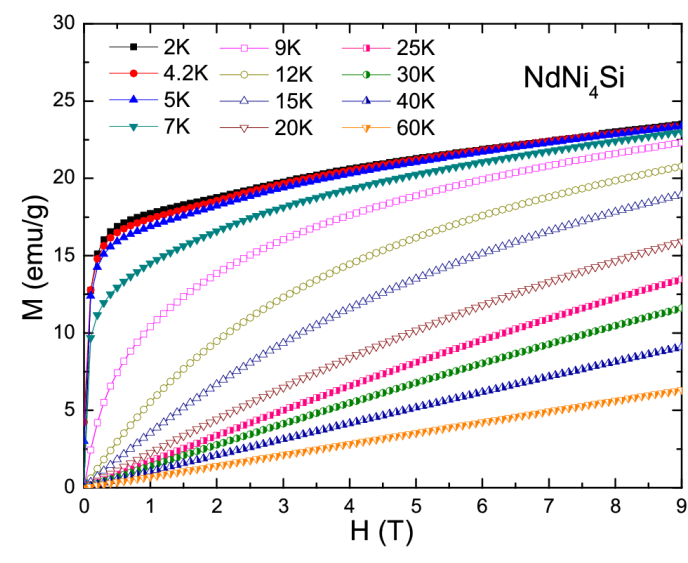

Fig. 1. The isothermal magnetization curves at selected temperatures up to $9 \mathrm{~T}$ for $\mathrm{NdNi}_{4} \mathrm{Si}$.

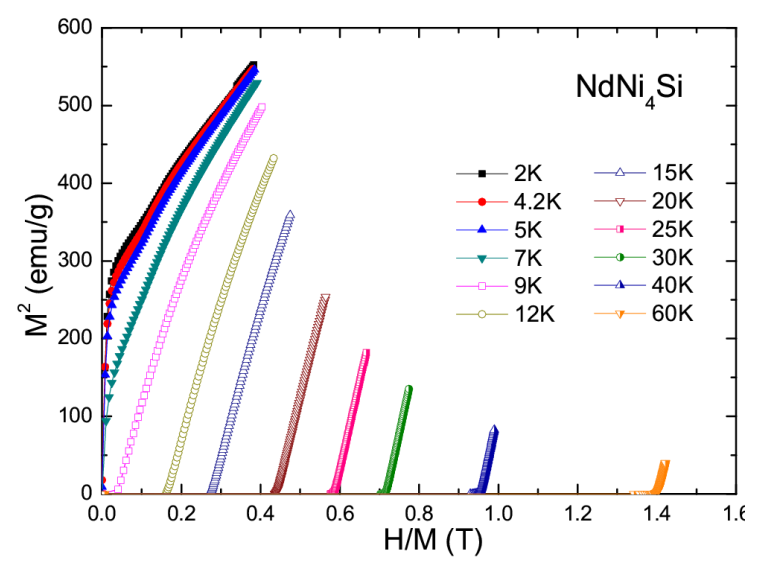

Fig. 2. Arrot plot for temperatures in the neighborhood of $T_{\mathrm{C}}$.

In order to investigate the MCE, which can be expressed as the adiabatic change in entropy upon the magnetic field application, we used the integrated Maxwell thermodynamical relation $[7,8]$ :

$$
\Delta S_{\mathrm{M}}(T, H)=\int_{H_{1}}^{H_{2}}\left(\frac{\delta M(T, H)}{\delta T}\right)_{H} \mathrm{~d} H .
$$

For magnetization isotherms measured at different constant temperatures the above relation can be approximated to the following expression $[9,10]$ :

$$
\begin{aligned}
& \Delta S_{\mathrm{M}}(T) \approx \frac{\mu_{0}}{\delta T}\left[\int_{0}^{H_{\max }} M(T+\delta T, H) \mathrm{d} H\right. \\
& \left.-\int_{0}^{H_{\max }} M(T, H) \mathrm{d} H\right],
\end{aligned}
$$

where $S, M, H$, and $T$ are the magnetic entropy, magnetization, applied magnetic field, and the temperature of the system, respectively. $\Delta S_{\mathrm{M}}$ can be regarded as a measure for the difference in area under two magnetization curves as shown in Fig. 1. It is pointed out that the magnitude of the magnetic entropy change and its dependence on temperature and magnetic field are strongly related to the nature of the corresponding magnetic phase transition. For ferromagnetic materials mainly the second order type of the magnetic phase transition is observed.

Figure 3 displays the temperature dependence of the magnetic entropy change for $\mathrm{NdNi}_{4} \mathrm{Si}$ under different external magnetic fields. The maximum $\Delta S_{\mathrm{M}}$ of $H=9 \mathrm{~T}$ reaches a quite large value of $9.2 \mathrm{~J} \mathrm{~kg}^{-1} \mathrm{~K}^{-1}$ around $T_{\mathrm{C}}$. The MCE is related to the second order Nd-Nd sublattice magnetic phase transition. In our previous paper we have shown that for $\mathrm{RNi}_{4} \mathrm{Si}$ compounds, the $T_{\mathrm{C}}$ values scale approximately with the de Gennes factor indicating that the coupling of the $\mathrm{R}-\mathrm{R}$ moments is due to the indirect exchange interactions via the conduction electrons (Ruderman-Kittel-Kasuya-Yosida (RKKY) interaction) [5]. The local magnetic moments of Ni atoms estimated from the linear muffin-tin orbital (LMTO) calculation for $\mathrm{GdNi}_{4} \mathrm{Si}$ was very small $\left(M_{\mathrm{Ni}}=0.03 \mu_{\mathrm{B}} /\right.$ atom $)$ and his contribution was neglected [11].

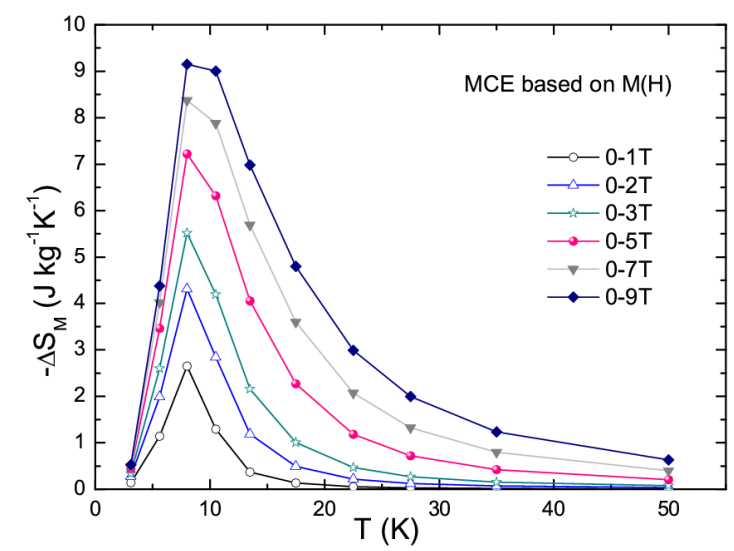

Fig. 3. Temperature variation of the magnetic entropy change determined from the magnetization curves for a field up to $9 \mathrm{~T}$.

The MCE can be also well determined from the specific heat $C_{p}(T)$ data as a function of temperature in constant magnetic fields. In the case of $C_{p}(T)$ the magnetic entropy change can be calculated by using the expression [9]:

$$
\Delta S_{\mathrm{M}}(T, H)=\int_{0}^{T}\left(\frac{C_{p}(T, H)-C_{p}(T, 0)}{T}\right) \mathrm{d} T,
$$

where $C_{p}(T, H)$ and $C_{p}(T, 0)$ are the values of the specific heat measured in a field $H$ and in zero field. Figure 4 shows the resulting MCE obtained from the $C_{p}(T)$ data in several selected magnetic fields. The maximum values of $\Delta S_{\mathrm{M}}$ around the magnetic ordering temperature for magnetic fields of $9,7,5$ and $3 \mathrm{~T}$ are 10.1, 9.1, 7.8, and $6 \mathrm{~J} \mathrm{~kg}^{-1} \mathrm{~K}^{-1}$, respectively and one can see that these values are in a good agreement with the values obtained from the isothermal magnetization. 


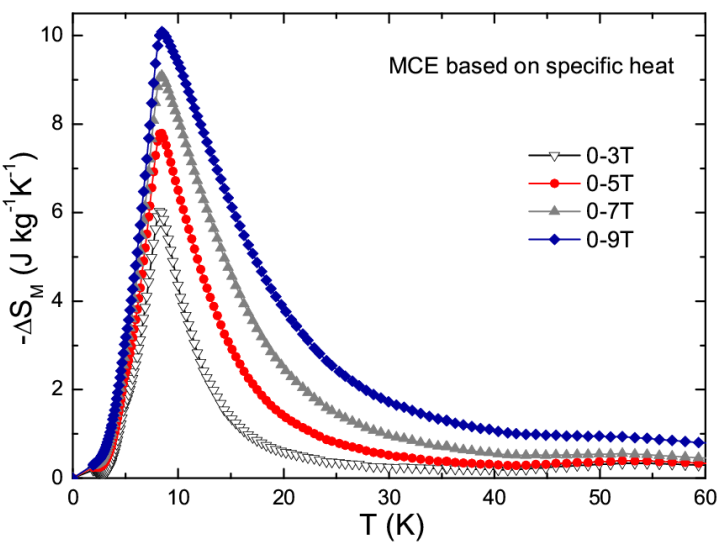

Fig. 4. Temperature variation of the magnetic entropy change obtained from the specific heat data for selected magnetic fields.

Another important parameter for MCE is the adiabatic temperature change $\Delta T_{\mathrm{ad}}$, which can also be obtained from the entropy calculated from $C_{p}(T)$ as a function of temperature in various magnetic fields. The isoentropic difference between two entropy curves gives $\Delta T_{\mathrm{ad}}[9]$ :

$$
\Delta T_{\mathrm{ad}}(T)_{\Delta H}=\left[T(S)_{H}-T(S)_{H=0}\right]_{S} .
$$

Figure 5 shows the temperature dependence of the adiabatic temperature change calculated for selected magnetic fields. For the fields change of $9,7,5,3 \mathrm{~T}$ the maximum values of $\Delta T_{\mathrm{ad}}$ are $6.6,6,4.5$, and $3.2 \mathrm{~K}$, respectively.

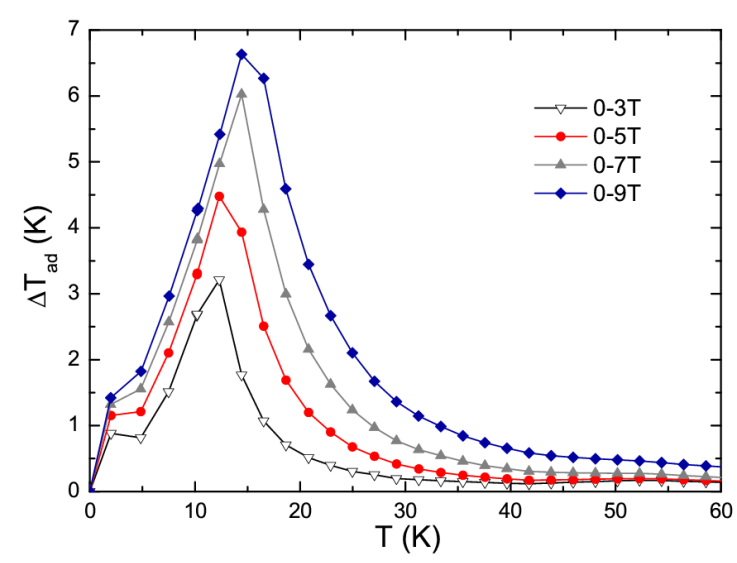

Fig. 5. Adiabatic temperature change as a function of temperature calculated from the specific heat data for $\mathrm{NdNi}_{4} \mathrm{Si}$ at selected magnetic fields.

One of the main criterions which should be fulfilled in magnetic refrigerators is the large magnetic entropy change and the large adiabatic temperature change. However, for the practical applicability in refrigeration an important quality parameter is the relative cooling power (RCP), which is a measure of the amount of the transferred heat $[12,13]$. RCP is a product of the maximum magnetic entropy change $\Delta S_{\mathrm{M}}$ and full width at half maximum for the $\Delta S_{\mathrm{M}}(T)$ curve and can be calculated using the formula: $\operatorname{RCP}(S)=-\Delta S_{\mathrm{M}}^{\max } \delta T_{\mathrm{FWHM}}$ [14], where $\delta T_{\mathrm{FWHM}}$ is the full width at half maximum. For $\mathrm{NdNi}_{4} \mathrm{Si}$ we have got $\operatorname{RCP}(S)=84 \mathrm{~J} \mathrm{~kg}^{-1}$ and analogically, for $\Delta T_{\mathrm{ad}}(T)$ curves $\operatorname{RCP}(T)=145 \mathrm{~K}^{2}$ in $H=7 \mathrm{~T}$.

\section{Conclusions}

We can conclude that our data obtained from specific heat and magnetization measurements reveal the attendance of $\mathrm{MCE}$ in $\mathrm{NdNi}_{4} \mathrm{Si}$ compound. For both types of measurements the magnitude of MCE is comparable. The values of the magnetic entropy change and the adiabatic temperature change indicates that MCE in this compound is quite large.

\section{Acknowledgments}

This work was supported by the funds of the National Science Centre as a research project no. N N507 219540 in years 2011-2013.

\section{References}

[1] V.K. Pecharsky, K.A. Gschneidner, Jr., Phys. Rev. Lett. 78, 4494 (1997).

[2] N.H. Duc, D.T. Kim Anh, P.E. Brommer, Physica B 319, 1 (2002).

[3] P.J. von Ranke, D.F. Grangeia, A. Caldas, N.A. de Oliveira, J. Appl. Phys. 93, 4055 (2003).

[4] P.J. von Ranke, M.A. Motta, D.F. Grangeia, A. Magnus, G. Carvalho, F.C.G. Gandra, A.A. Coelho, A. Caldas, N.A. de Oliveira, S. Gama, Phys. Rev. B 70, 134428 (2004).

[5] M. Falkowski, B. Andrzejewski, A. Kowalczyk, J. Alloys Comp. 442, 155 (2007).

[6] M. Falkowski, M. Reiffers, M. Zapotoková, A. Kowalczyk, T. Toliński, E. Gažo, Acta Phys. Pol. A 115, 126 (2009).

[7] V.K. Pecharsky, K.A. Gschneidner, Jr., J. Magn. Magn. Mater. 200, 44 (1999).

[8] A.M. Tishin, Y.I. Spichkin, The Magnetocaloric Effect and Its Applications, IOP Publishing, Ltd., Bristol 2003.

[9] V.K. Pecharsky, K.A. Gschneidner, Jr., J. Appl. Phys. 86, 565 (1999).

[10] K.A. Gschneidner, Jr., V.K. Pecharsky, A.O. Tsokol, Rep. Prog. Phys. 68, 1479 (2005).

[11] A. Kowalczyk, A. Szajek, M. Falkowski, G. Chełkowska, J. Magn. Magn. Mater. 305, 348 (2006).

[12] K.A. Gschneidner, Jr., V.K. Pecharsky, Ann. Rev. Mater. Sci. 30, 387 (2000).

[13] K.A. Gschneidner, Jr., V.K. Pecharsky, in: Intermetallic Compounds - Principles and Practica, Eds. J.H. Westbrook, R.L. Fleischer, Vol. 3, Wiley, New York 2001.

[14] B. Li, J. Du, W.J. Ren, W.J. Hu, Q. Hang, D. Li, Z.D. Hang, Appl. Phys. Lett. 92, 242504 (2008). 